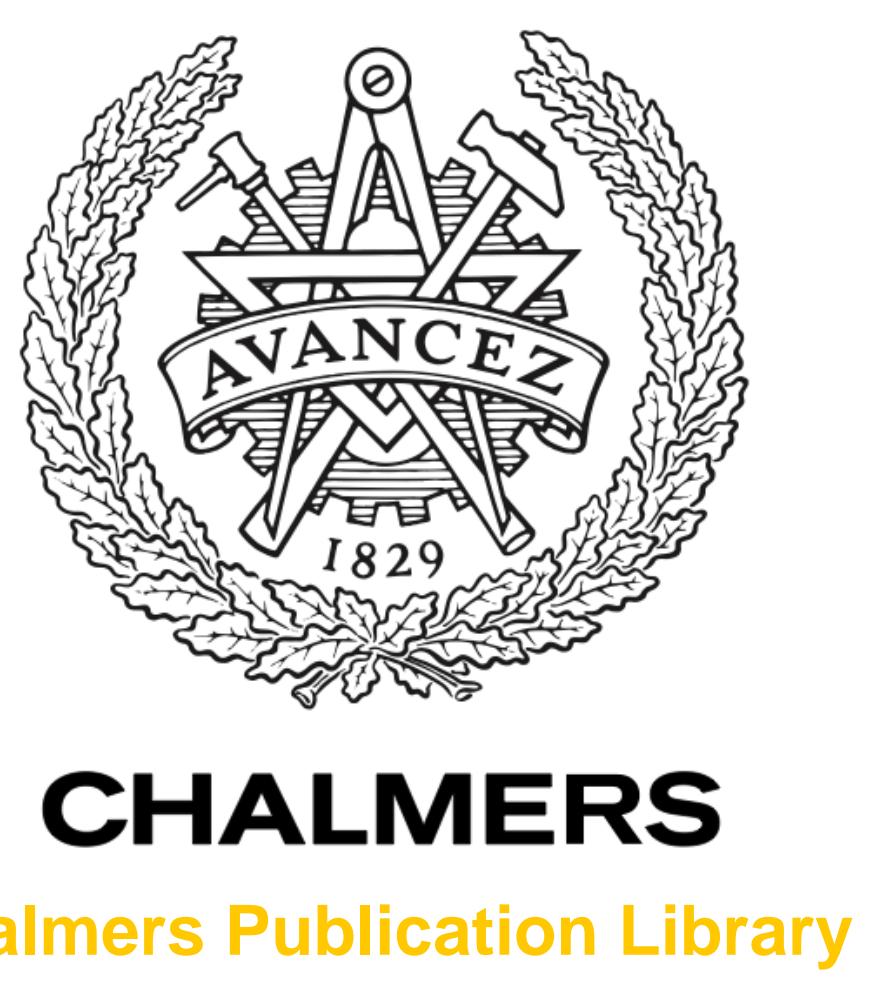

\title{
Experimental comparison of PS-QPSK and LDPC-coded PM-QPSK with equal spectral efficiency in WDM transmission
}

This document has been downloaded from Chalmers Publication Library (CPL). It is the author's version of a work that was accepted for publication in:

2014 European Conference on Optical Communication, ECOC 2014; Cannes; France; 21 September 2014 through 25 September 2014

\begin{abstract}
Citation for the published paper:
Eriksson, T. ; Johannisson, P. ; Agrell, E. et al. (2014) "Experimental comparison of PSQPSK and LDPC-coded PM-QPSK with equal spectral efficiency in WDM transmission". 2014 European Conference on Optical Communication, ECOC 2014; Cannes; France; 21 September 2014 through 25 September 2014 pp. Art. no. 6964035.
\end{abstract}

http://dx.doi.org/10.1109/ECOC.2014.6964035

Downloaded from: http://publications.lib.chalmers.se/publication/209245

Notice: Changes introduced as a result of publishing processes such as copy-editing and formatting may not be reflected in this document. For a definitive version of this work, please refer to the published source. Please note that access to the published version might require a subscription. 


\title{
Experimental Comparison of PS-QPSK and LDPC-Coded PM-QPSK with Equal Spectral Efficiency in WDM Transmission
}

\author{
Tobias A. Eriksson ${ }^{(1)}$, Pontus Johannisson ${ }^{(1)}$, Erik Agrell ${ }^{(2)}$, Peter A. Andrekson ${ }^{(1)}$, Magnus Karlsson ${ }^{(1)}$ \\ (1) Department of Microtechnology and Nanoscience. ${ }^{(2)}$ Department of Signals and Systems. \\ Chalmers University of Technology, SE-412 96, Gothenburg, Sweden. Email: tobias.eriksson@chalmers.se
}

\begin{abstract}
We compare QPSK with a rate 0.75 LDPC-code and PS-QPSK, which have the same spectral efficiency, in long-haul WDM transmission. We show that LDPC-coded PM-QPSK can achieve up to $98 \%$ increased transmission distance over uncoded PS-QPSK at BER $=10^{-3}$.
\end{abstract}

\section{Introduction}

Four-dimensional (4D) modulation formats have received a lot of research attention in the last few years and were first introduced to the fiber-optic community in 2009 by Bülow ${ }^{1}$ as well as Agrell and Karlsson $^{2}$. Polarization-switched quadrature phase-shift keying (PS-QPSK) was found to be the most powerefficient 4D modulation format ${ }^{2}$ and in experiments it was shown that PS-QPSK can achieve around $30 \%$ increased transmission distance over PM-QPSK at the same bit rate ${ }^{3,4}$. However, PS-QPSK has $25 \%$ lower spectral efficiency (SE) compared to PM-QPSK. PM-QPSK and PS-QPSK have been compared with forward-error correction (FEC) codes based on ReedSolomon (RS) and low-density parity check (LDPC) ${ }^{5}$. It was found that PM-QPSK with a sufficiently powerful code can achieve better sensitivity compared to PS-QPSK, when they are compared with different overhead to achieve equal bandwidth and bit rate after the FEC ${ }^{5}$. This can be understood from the fact that PS-QPSK can be expressed as a single-parity check code on PM-QPSK and that PS-QPSK is a subset of PM-QPSK. In general, a better performing system can be found by increasing the number of constellation points of the modulation format and utilizing the increased SE for a more powerful code. However, this will lead to a more complex system and it should be noted that PS-QPSK can be decoded on a symbol-bysymbol basis requiring similar receiver and transmitter complexity as PM-QPSK. Most demonstrations of realtime coherent receivers exclude the FEC circuits with a few exceptions ${ }^{6}$, leading to the question of acceptable complexity of the decoder. Several fiber-optic transmission experiments utilizing LDPC codes have been demonstrated with for instance 16-QAM ${ }^{7}, 3$-ary phase-shift keying ${ }^{8}, \mathrm{PM}-\mathrm{QPSK}^{9}$, and 24-dimensional extended Golay coded modulation ${ }^{10}$.

In this paper, we compare PM-QPSK, PS-QPSK, and PM-QPSK with a rate 0.75 LDPC (PM-LDPCQPSK) code in wavelength-division multiplexed (WDM) transmission over long-haul transmission distances at different launch powers. We compare the three systems at equal symbol rate, giving PS-QPSK and PMLDPC-QPSK the same SE and bit rate. We show that it is possible to achieve up to $98 \%$ increased transmission distance for PM-LDPC-QPSK over PS-QPSK.

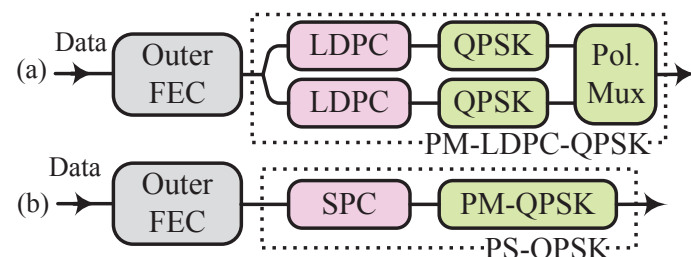

Fig. 1. Illustration of the two systems compared in this paper. (a) QPSK with a rate 0.75 LDPC code and polarization multiplexing. (b) PS-QPSK, which can be interpreted as an single-parity check (SPC) code on PM-QPSK. The outer FEC code (gray) was not implemented.

\section{LDPC-Coded Modulation}

The two systems compared in this paper are illustrated in Fig. 1 . We apply a rate 0.75 LDPC code with a block length of 64,800 from the DVB-S2 standard ${ }^{11}$ on QPSK at a symbol rate of $28 \mathrm{Gbaud}$. We then use polarization multiplexing to achieve the same SE and bandwidth as 28 Gbaud PS-QPSK. This case has been studied in simulations over an additive white Gaussian noise (AWGN) channel using the same LDPC code as in this paper and it was found that below a BER of $\sim$ $2.5 \times 10^{-2}$, the LDPC-coded QPSK outperforms PSQPSK in terms of required OSNR ${ }^{12}$. We assume that an outer FEC code is used, since the BER floor of this LDPC code is not known for the nonlinear fiber channel and it is unfeasible to measure such low BERs with offline processing. It should be noted though that many FEC codes assume independent data to have optimal functionality and with the outer code applied, the bits passed to the LDPC encoder are no longer independent. The use of an outer RS code and an inner LPDC code has been demonstrated with real-time encoders and decoders for an on-off keying system, showing that such a system is feasible ${ }^{13}$.

\section{Experimental Setup}

The experimental setup using 7 WDM channels is shown in Fig. 2. As light sources, 7 distributed feedback lasers (DFB) with linewidths of $\sim 100 \mathrm{kHz}$ and spaced $50 \mathrm{GHz}$ apart are used. For PM-QPSK and PM-LDPC-QPSK, two I/Q-modulators, one for the 3 odd channels and one for the 4 even channels, are used. The WDM channels are combined using an optical interleaver (IL). For PS-QPSK, the two I/Qmodulators are placed to modulate data in two orthogonal polarization states and all WDM channels are 


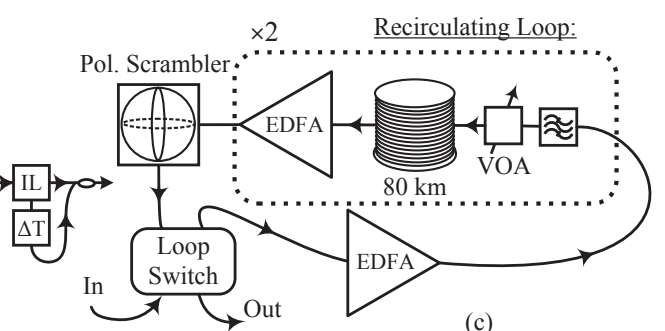

Fig. 2. Experimental setup showing (a) the transmitter for PM-QPSK as well as PM-LDPC-QPSK. The optical interleaver (IL) combines the even and odd channels and are followed by a polarization multiplexing emulation stage. (b) The transmitter for PS-QPSK. An IL is used to decorrelate the even and odd channels. (c) The recirculating loop with two spans of $80 \mathrm{~km}$ SMF and a polarization scrambler. Before the

first, span an optical bandpass filter with $4 \mathrm{~nm}$ bandwidth is used and before the second span, an optical processor is used as both bandpass filter and for gain equalization. The variable optical attenuators (VOAs) are used to set the optical launch power for each span.

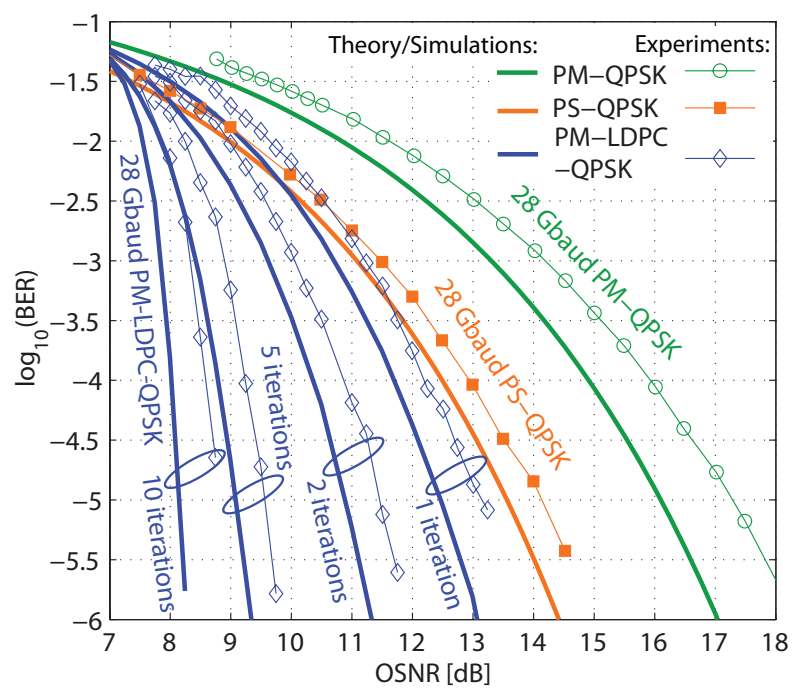

Fig. 3. Back-to-back results showing BER as a function of OSNR $(0.1 \mathrm{~nm})$ for experimentally measured PM-QPSK (green circles),

PS-QPSK (orange squares) and PM-LDPC-QPSK (blue diamonds).

Theoretical predictions for PM-QPSK (solid green line) and

PS-QPSK (solid orange line) as well as simulation results with AWGN as the only impairment for PM-LDPC-QPSK with 1, 2, 5 and 10 iterations in the decoder (solid blue lines).

modulated using this transmitter setup and decorrelated after the transmitter using an IL and a time delay for the odd channels.

The I/Q-modulators are driven by a 28 Gbaud binary pattern. For PM-QPSK and PS-QPSK, the patterns used were pseudorandom binary sequences with length $2^{15}-1$ (PRBS15) and for PM-LDPC-QPSK, the pattern generator was programmed with LDPC codewords constructed from concatenated PRBS15. The total length of the LDPC-coded sequence was $4,114,800$ symbols. For PS-QPSK, one of the 4 patterns are encoded as an XOR-operation on the other 3.

The signals were propagated in a recirculating loop consisting of two spans of $80 \mathrm{~km}$ of single-mode fiber. Before the first span, an optical bandpass filter with $4 \mathrm{~nm}$ bandwidth was used and preceding the second span was an optical processor performing both bandpass filtering and gain equalization. The gain was equalized over the average power per carrier over all loop round-trip times and the difference was less than $2 \mathrm{dBm}$. The difference in power per carrier launched to the loop was less than $0.2 \mathrm{dBm}$.

The receiver consisted of a polarization-diverse optical hybrid with a free-running external cavity laser with $\sim 300 \mathrm{kHz}$ linewidth as local oscillator. The received optical signal was amplified using an EDFA and an optical bandpass filter with $0.9 \mathrm{~nm}$ bandwidth was used to suppress out-of-band noise and attenuate the neighboring WDM channels. All measurements were performed on the center channel. The electrical signals from the hybrid were sampled using a $100 \mathrm{GS} / \mathrm{s}$ oscilloscope with $33 \mathrm{GHz}$ bandwidth.

We use a conventional blind receiver structure with dispersion compensation in the frequency domain and polarization demultiplexing and adaptive equalization using the constant modulus algorithm (CMA) for PMQPSK and PM-LDPC-QPSK and the modified CMA ${ }^{14}$ for PS-QPSK. Further, frequency estimation based on the Fourier transform and phase estimation based on Viterbi-Viterbi algorithm are used. For PM-LDPCQPSK, the received codewords are decoded using the message-passing algorithm with $1,2,5$, or 10 iterations.

\section{Experimental Results}

Fig. 3 shows the measured back-to-back BER as a function of the OSNR $(0.1 \mathrm{~nm})$ for 28 Gbaud PMQPSK, 28 Gbaud PS-QPSK, and 28 Gbaud PMLDPC-QPSK with 1, 2, 5, and 10 iterations in the decoder for a single channel with the interleaver filter included in the transmitter. Also shown are the theoretical predictions for PM-QPSK and PS-QPSK and simulation results with AWGN as the only impairment for PM-LDPC-QPSK with 1, 2, 5, and 10 iterations. If we compare the formats at $\mathrm{BER}=10^{-3}$, the implementation penalty is $0.9 \mathrm{~dB}$ for PM-QPSK and $0.4 \mathrm{~dB}$ for PS-QPSK. For PM-LDPC-QPSK, we see around $0.5 \mathrm{~dB}$ implementation penalty for the three different iterations in the decoder. The linear crosstalk when the two nearest neighboring channels were present was less than $0.5 \mathrm{~dB}$ for all cases.

The measured sensitivity gain at $B E R=10^{-3}$ was 2.7 dB for PS-QPSK over PM-QPSK and PM-LDPCQPSK has a measured sensitivity gain over PS-QPSK of $0.3 \mathrm{~dB}, 1.4 \mathrm{~dB}, 2.6 \mathrm{~dB}$, and $3.1 \mathrm{~dB}$ for 1, 2, 5, and 10 iterations in the decoder, respectively. The 


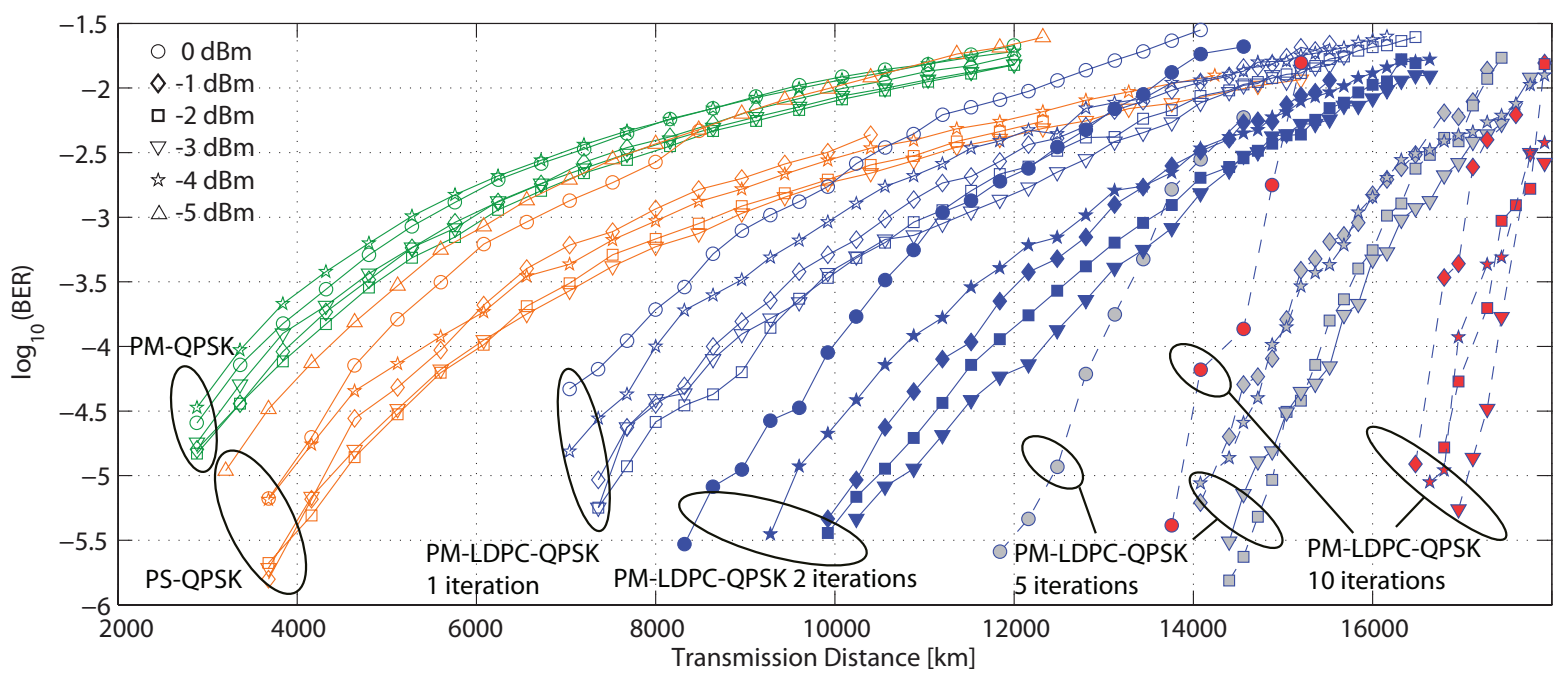

Fig. 4. Experimental transmission results showing BER as a function of distance for the center channel of the $7 \times 28 \mathrm{Gbaud}$ WDM channels for PM-QPSK (green), PS-QPSK (orange), and PM-LDPC-QPSK with one iteration (open blue), two iterations (filled blue), five iterations (blue line, gray symbols), and 10 iterations (blue line, red symbols). The different symbols represent different launch power per channel.

corresponding numbers comparing the theoretical prediction for PS-QPSK and the simulation results for PM-LDPC-QPSK are $0.4 \mathrm{~dB}, 1.5 \mathrm{~dB}, 2.7 \mathrm{~dB}$ and $3.2 \mathrm{~dB}$. Using 5 iterations, the experimental sensitivity gain over uncoded PM-QPSK is $5.3 \mathrm{~dB}$.

The experimental WDM transmission results for 7 $\times 28$ Gbaud measured on the center channel for all formats are shown in Fig. 4. It should be noted that depending on which outer FEC code that is used, the formats should be compared at different BER levels. However, since we do not implement the outer FEC, we choose to compare the formats at $B E R=10^{-3}$, which is in the region were many FEC codes can operate. We investigate the formats at different launch powers and the optimal launch power per channel was $-2 \mathrm{dBm}$ for PM-QPSK and $-3 \mathrm{dBm}$ for PS-QPSK. For PM-LDPCQPSK the optimal power was $-3 \mathrm{dBm}$ independently of the number of iterations. We observe a large penalty for going to higher launch powers, most prominent at 5 iterations were the transmission distance is reduced by $17 \%$ when going from $-3 \mathrm{dBm}$ launch power to $0 \mathrm{dBm}$.

PM-QPSK can be transmitted up to $6,100 \mathrm{~km}$ and PS-QPSK up to $8,900 \mathrm{~km}$, which corresponds to an increase of $46 \%$. PM-LDPC-QPSK can be transmitted up to $11,400 \mathrm{~km}$ with 1 iteration, $13,900 \mathrm{~km}$ with 2 iterations, $16,300 \mathrm{~km}$ with 5 iterations, and $17,600 \mathrm{~km}$ with 10 iterations. Using 10 iterations, the transition between a BER that is lower than what we can measure with offline processing and high BER occurs over a narrower distance range than for fewer iterations. The increase in transmission reach for PM-LDPCQPSK over PS-QPSK is $28 \%, 56 \%, 83 \%$, and $98 \%$ for 1, 2, 5, and 10 iterations, respectively.

\section{Conclusions and Discussion}

We have compared PM-QPSK with a rate 0.75 LDPC code and PS-QPSK at 28 Gbaud, yielding the same bit rate and spectral efficiency. We performed longhaul WDM transmission with $7 \times 28$ Gbaud channels. We have shown transmission of PM-LDPC-QPSK of up to $17,600 \mathrm{~km}$, which is $98 \%$ longer than PS-QPSK using a maximum of 10 iterations in the LDPC decoder. We leave the question of how much extra complexity, power consumption, and latency the LDPC system will have in a real-time implementation open. It should be noted that even though the PM-LDPC-QPSK outperforms PS-QPSK, the latter has similar hardware complexity as PM-QPSK.

\section{References}

[1] H. Bülow et al., "Polarization QAM Modulation (POL-QAM) for Coherent Detection Schemes", Proc. OFC'09, OWG2 (2009).

[2] E. Agrell \& M. Karlsson, "Power-Efficient Modulation Formats in Coherent Transmission Systems", J. Lightw. Tech., 27, 5115 (2009).

[3] D. S. Millar et al., "Generation and Long-Haul Transmission of Polarization-Switched QPSK at $42.9 \mathrm{~Gb} / \mathrm{s}$ ", Opt. Exp., 19, 9296 (2011).

[4] M. Sjödin et al., "Transmission of PM-QPSK and PS-QPSK with Different Fiber Span Lengths", Opt. Exp., 20, 7544 (2012).

[5] B. Krongold et al., "Comparison Between PS-QPSK and PDMQPSK With Equal Rate and Bandwidth", IEEE Photon. Technol. Lett., 24, 203 (2012).

[6] K. Fukuchi et al., "112Gb/s Optical Transponder with PM-QPSK and Coherent Detection Employing Parallel FPGA-based RealTime Digital Signal Processing, FEC and $100 \mathrm{GbE}$ Ethernet Interface", Proc. ECOC'10, Tu.5.A.2 (2010).

[7] D. G. Foursa et al., "44.1 Tb/s Transmission over $9,100 \mathrm{~km}$ Using Coded Modulation Based on 16QAM Signals at $4.9 \mathrm{bits} / \mathrm{s} / \mathrm{Hz}$ Spectral Efficiency", Proc. ECOC'13, PD3.E.1, (2013).

[8] F. Buchali et al., " $550 \mathrm{~Gb} / \mathrm{s}$ WDM Transmission of 32 Gbaud DP-3PSK over 36,000 km Fiber with Spatially Coupled LDPC Coding", Proc. OFC'14, W1A.1, (2014).

[9] C. Xie et al., "Transmission of 130-Gb/s PDM-QPSK over 5,760 $\mathrm{km}$ with Co-Propagating $10-\mathrm{Gb} / \mathrm{s}$ OOK Channels in DispersionManaged NZDSF Spans with Soft-Decision LDPCC Coding", Proc. OFC'13, JW2A.40, (2013).

[10] D. S. Millar et al., "Experimental Demonstration of 24-Dimensional Extended Golay Coded Modulation with LDPC", Proc. OFC'14, M3A.5, (2014).

[11] Digital Video Broadcasting (DVB-S2), ETSI, V1.2.1, (2009).

[12] T. Pfau et al., "Comparison of Bandwidth Expansion Methods for Optical Transmission Systems", Proc. OECC'12, 5B4-1 (2012).

[13] T. Mizuochi et al., "112Gb/s Optical Transponder with PM-QPSK and Coherent Detection Employing Parallel FPGA-based RealTime Digital Signal Processing, FEC and 100GbE Ethernet Interface", IEEE Photon. Technol. Lett., 21, 1302 (2009).

[14] P. Johannisson et al., "Modified Constant Modulus Algorithm for Polarization-Switched QPSK", Opt. Exp. 19, 7734 (2011). 